Does capillary evaporation limit the accessibility of nonaqueous electrolytes to the ultrasmall pores of carbon electrodes?

Kun Liu, Pengfei Zhang, and Jianzhong Wu

Citation: J. Chem. Phys. 149, 234708 (2018); doi: 10.1063/1.5064360

View online: https://doi.org/10.1063/1.5064360

View Table of Contents: http://aip.scitation.org/toc/jcp/149/23

Published by the American Institute of Physics

\section{PHYSICS TODAY}

WHITEPAPERS
ADVANCED LIGHT CURE ADHESIVES

READ NOW

Take a closer look at what these environmentally friendly adhesive systems can do
PRESENTED BY

Q MASTERBOND 


\title{
Does capillary evaporation limit the accessibility of nonaqueous electrolytes to the ultrasmall pores of carbon electrodes?
}

\author{
Kun Liu, ${ }^{1}$ Pengfei Zhang, ${ }^{2}$ and Jianzhong $\mathrm{Wu}^{1, \mathrm{a})}$ \\ ${ }^{1}$ Department of Chemical and Environmental Engineering, University of California, Riverside, \\ California 92521, USA \\ ${ }^{2}$ Division of Chemistry and Chemical Engineering, California Institute of Technology, Pasadena, \\ California 91125, USA
}

(Received 3 October 2018; accepted 26 November 2018; published online 21 December 2018)

\begin{abstract}
Porous carbons have been widely utilized as electrode materials for capacitive energy storage. Whereas the importance of pore size and geometry on the device performance has been well recognized, little guidance is available for identification of carbon materials with ideal porous structures. In this work, we study the phase behavior of ionic fluids in slit pores using the classical density functional theory. Within the framework of the restricted primitive model for nonaqueous electrolytes, we demonstrate that the accessibility of micropores depends not only on the ionic diameters (or desolvation) but also on their wetting behavior intrinsically related to the vapor-liquid or liquid-liquid phase separation of the bulk ionic systems. Narrowing the pore size from several tens of nanometers to subnanometers may lead to a drastic reduction in the capacitance due to capillary evaporation. The wettability of micropores deteriorates as the pore size is reduced but can be noticeably improved by raising the surface electrical potential. The theoretical results provide fresh insights into the properties of confined ionic systems beyond electric double layer models commonly employed for rational design/selection of electrolytes and electrode materials. Published by AIP Publishing. https://doi.org/10.1063/1.5064360
\end{abstract}

\section{INTRODUCTION}

Capacitive energy storage hinges on electrochemical adsorption/desorption of ionic species at the surface of porous electrodes that are typically made of various forms of activated carbons. ${ }^{1,2}$ The energy storage capacity can be improved by increasing the electric double layer (EDL) capacitance, which is linearly proportional to the surface area, and/or by using nonaqueous electrolytes (viz., organic electrolytes or room-temperature ionic liquids) with wide operating potential windows (OPW). The importance of pore size and shape on capacitive energy storage has been well documented. ${ }^{3,4}$ The customary opinion is that mesoporous carbons, with the average pore size larger than $2 \mathrm{~nm}$, are ideally suited for the electrode material, while micropores make no significant contributions to energy storage because they are not easily accessible to solvated ions and the ionic motions in such pores may be severely compromised. ${ }^{5}$ Recently, a number of experimental and theoretical studies indicate that an anomalous increase in the EDL capacitance can be achieved by matching the pore size with the diameters of ionic species ${ }^{6}$ and that ion diffusion in micropores can be an order of magnitude faster than that in the bulk. ${ }^{7}$ However, it has been shown by Monte Carlo (MC) simulation that a first-order phase transition may occur in porous electrodes when the pore size is comparable to the ion size.$^{8-10}$ Recent molecular dynamics (MD) simulation also indicates that capillary evaporation may

a)Author to whom correspondence should be addressed: jwu@engr.ucr.edu take place in 1-ethyl-3-methylimidazolium tetrafluoroborate ([EMIM] $\left.\left[\mathrm{BF}_{4}\right]\right)$ confined between solvophobic sheets. ${ }^{11}$

Experimental verification of the pore-size effects on EDL capacitance has been elusive because activated carbons typically do not have a well-defined pore structure. The problem is further complicated by the imprecise characterization of porous materials in terms of the surface areas and the pore size distributions. For example, the Brunauer-Emmett-Teller (BET) adsorption isotherms are routinely used to determine the specific surface areas of porous material, but it only works for adsorbents with large pores because it assumes uniform adsorption of gas molecules on a flat surface without lateral interactions. For micropores (pore size $<2 \mathrm{~nm}$ ), the BET analysis describes only an apparent surface area instead of a true geometrically accessible area. The BET and geometrical surface areas may differ by orders of magnitude. ${ }^{12}$ It has been shown that the capacitance per unit area is virtually independent of the average pore size for an organic electrolyte, $\left(\mathrm{C}_{2} \mathrm{H}_{5}\right)_{4} \mathrm{NBF}_{4} /$ acetonitrile, in electrodes made of carbon monoliths with narrow pore size distributions. ${ }^{13}$ Furthermore, carbon electrodes exhibiting a large area-specific capacitance are not necessarily correlated with those with a high gravimetric capacitance. ${ }^{14}$ Nevertheless, a number of theoretical investigations of ionic liquids in idealized pores unequivocally predict a maximized capacitance when the pore size is comparable to the ion diameters, albeit the oscillatory dependence of the capacitance on the pore size has been subjected to rather different interpretations. ${ }^{15-18}$ While various effects may contribute to the oscillatory variation of the capacitance as a function of the pore size, the discrepancy between theory and the experiment may also arise from the 
accessibility of micropores to ionic species. To our knowledge, none of previous investigations of EDL capacitance account for the accessibility or the phase behavior of ionic liquids in small pores at conditions pertinent to energy storage.

Conventionally the accessibility of ionic fluids to the micropores of a carbon electrode is often loosely defined in terms of the pore geometry; viz., a pore is accessible to solvated or bare ions if its size is larger than the ion diameter. While the geometric constraint for the accessibility of individual ions is rather intuitive, such a definition of pore accessibility can easily be misleading because it assumes molecules as rigid bodies and ignores multi-body correlations or collective effects. Micropores larger than the ionic diameters may not make a significant contribution to energy storage if the ion density inside the pore is exceedingly low. Boukhalfa $e t$ al. used in situ small angle neutron scattering (SANS) to study the electroadsorption of organic ions in carbon pores of different sizes. They observed incomplete wetting of the smallest carbon pores by deuterated acetonitrile and enhanced ion sorption in subnanometer pores under the applied potential. This behavior may explain the characteristic butterfly wing shape of the cyclic voltammetry curve of specific capacitance in electrical double layer capacitors. ${ }^{19}$ The low-density state may be introduced by either vapor-liquid or liquid-liquid separation of the ionic fluid inside the pore. Unlike the size effects, phase transitions are collective phenomena depending not only on the interactions of ionic species with the pore surface and with themselves but also on the thermodynamic conditions. ${ }^{20}$ Although the wettability of micropores has been routinely discussed in the literature and, clearly, it plays an important role in commercial applications of porous electrodes, substantially less attention has been given to the phase behavior of ionic liquids and organic electrolytes under confinement. Previous studies on the pore-size effects on electrode performance are mostly focused on the EDL capacitances and the ionic distributions without an explicit consideration of possible phase transitions. ${ }^{3}$

Phase transitions in inhomogeneous simple fluids have been reasonably well understood. ${ }^{21}$ However, much less is known about how the phase behavior of ionic systems is influenced by confinement, especially under conditions whereby ionic fluids in micropores are subject to an external electrical potential. The long-range Coulomb interactions make the critical behavior and phase transitions in ionic fluids substantially more complicated than those corresponding to neutral systems. ${ }^{22}$ In comparison to the bulk systems, theoretical studies of the phase behavior of ionic liquids under confinement are necessarily even more difficult because they must account for the density inhomogeneity and be consistent with the existing results in the bulk limit.

Previous studies on the phase behavior of ionic liquids in the bulk are largely based on the restricted primitive model (RPM), in which ions are described as charged hard spheres of the same size and absolute valence. ${ }^{23}$ RPM has been widely used to represent the properties of both aqueous and organic electrolyte solutions, molten salts, as well as room-temperature ionic liquids. ${ }^{24}$ Although the model is much oversimplified to quantitatively reproduce the thermodynamic properties of any specific electrolyte, it incorporates key features of ionion interactions, namely, the excluded volume effects and the Coulomb potential, which are essential to describe the unique properties of ionic systems. As a result, RPM is routinely used to study the phase behavior of ionic systems including liquid-liquid equilibrium in organic electrolytes ${ }^{25,26}$ and wetting transitions of ionic liquids. ${ }^{27,28}$

Classical density functional theory (DFT) represents one of the most efficient theoretical tools to study phase transitions in confined fluids. ${ }^{29,30}$ Previously, different versions of DFT had been applied to describe the vapor-liquid coexistence of ionic fluids confined in slit pores. ${ }^{31,32}$ Within the framework of RPM for ionic systems, DFT predicts that confinement leads to a narrowed vapor-liquid coexistence region and reduces both the critical temperature and the critical density as the pore width decreases. It was found that an explicit consideration of association between oppositely charged ions reduces the critical temperature but does not change the qualitative behavior of the vapor-liquid-like phase diagram of confined ionic fluids. Similar predictions were made for the same ionic model in a cylindrical pore using the fieldtheoretical variational approach ${ }^{33}$ and in a disordered porous matrix using the method of collective variables. ${ }^{34,35}$ Consistent with MC simulations, ${ }^{8,9,36}$ these theoretical methods predict that the vapor-liquid coexistence region gets narrower as the pore width or porosity decreases. However, opposite trends were observed on the dependence of capillary evaporation on the electrostatic potential. While MC simulation shows that an ionic liquid in narrow pores can be stabilized by applying a surface electrical potential, classical DFT predicts destabilizing effects, suggesting that the theoretical results are sensitive to the formulation of the free-energy functional.

In this work, we study the wettability of nonaqueous electrolytes in the slit pores of carbon electrodes using a new version of classical DFT. RPM is used as a unified model to represent both vapor-liquid and liquid-liquid phase separations in ionic liquids and in organic electrolytes, respectively. Special attention is given to the effects of pore size and surface electrical potential on capillary evaporation and its implications to the properties of ionic fluids in the micropores of activated carbons important for capacitive energy storage. We find that capillary evaporation becomes more likely to occur as the pore width decreases and that the critical surface electrical potential exhibits a non-monatomic dependence on the pore size. The theoretical results offer fresh insights into the accessibility of ionic fluids to the ultrasmall pores of activated carbons important for the rational design and optimization of porous structure for energy storage.

\section{THE IONIC LIQUID MODEL AND THEORY}

\section{A. The restricted primitive model}

The restricted primitive model (RPM) assumes that cations and anions have the same diameter $\left(\sigma_{+}=\sigma_{-}=\sigma\right)$ and valence $\left(Z_{+}=-Z_{-}=1\right)$. In dimensionless units, the pair potential between two ionic species is given by 


$$
\beta u_{i j}(r)= \begin{cases}\infty, & r<\sigma_{i j} \\ Z_{i} Z_{j} l_{\mathrm{B}} / r, & r \geq \sigma_{i j},\end{cases}
$$

where $r$ is the center-center distance between ions $i$ and $j$, $\sigma_{i j}=\left(\sigma_{i}+\sigma_{j}\right) / 2, \beta=1 / k_{B} T$, and $l_{B}=\beta e^{2} /\left(4 \pi \epsilon_{0} \epsilon\right)$ is the Bjerrum length. As usual, $k_{B}$ is the Boltzmann constant, $T$ is the absolute temperature, $e$ is the unit charge, $\epsilon_{0}$ is the vacuum permittivity, and $\epsilon$ is the relative permittivity of the dielectric background.

In a slit-like pore of width $H$, each ion experiences an external energy along the normal direction of the planar walls positioned at $z=0$ and $z=H$,

$$
V_{i}(z)=V_{i}^{H W}(z)+V_{i}^{C}(z),
$$

where $V_{i}^{H W}(z)$ represents the hard-wall potential

$$
V_{i}^{H W}(z)= \begin{cases}\infty, & z<\sigma_{i} / 2 \text { or } z>H-\sigma_{i} / 2 \\ 0, & \text { otherwise }\end{cases}
$$

and $V_{i}^{C}(z)$ is the electrical potential due to the surface charge of the planar walls

$$
\beta V_{i}^{C}(z)=-2 \pi l_{B} Z_{i} H Q / e,
$$

where $Q$ is the surface charge density for each wall.

Figure 1 shows schematically that an ionic fluid in the bulk may exist either as a liquid or as a vapor (in the context of organic electrolytes, two liquid states of different ion densities) in a slit pore. For an ionic fluid with only one type of cations and one type of anions, the phase behavior in the bulk is typically described in terms of reduced temperature $T^{*}=4 \pi \epsilon \epsilon_{0} \sigma /\left(\beta e^{2}\right)=\sigma / l_{B}$ and reduced overall density $\rho^{*}=\left(\rho_{+}+\rho_{-}\right) \sigma^{3}$, where $\rho_{+}$and $\rho_{-}$are the number densities of cations and anions. For the ionic fluid in a slit pore, the phase behavior depends on thermodynamic conditions in the bulk as well as on the pore width and the surface charge or the surface electrical potential (i.e., voltage). Throughout this work, the pore width and the distance from a slit surface are denoted as $H^{*}=H / \sigma$ and $z^{*}=z / \sigma$, respectively. The dimensionless electric potential is defined as $\psi^{*}=\beta \psi e$, and the dimensionless charge density at the wall is $Q^{*}=Q \sigma^{2} / e$.

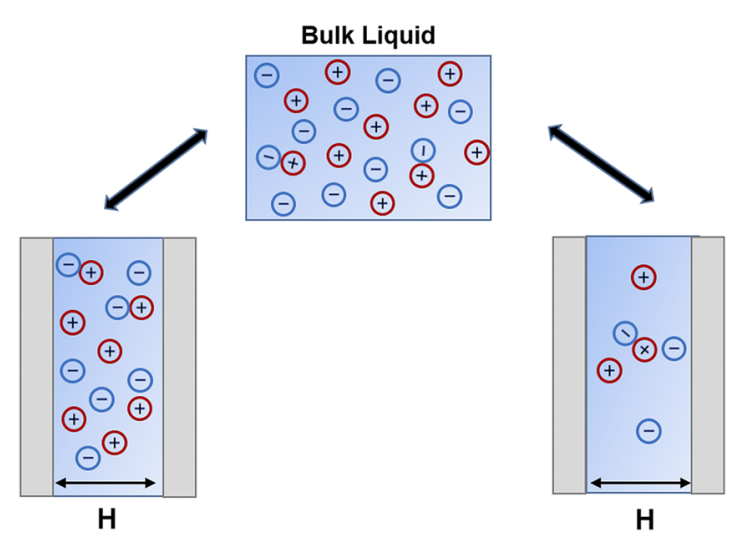

FIG. 1. Schematic picture of the model ionic liquid in a slit pore. The bulk liquid is in equilibrium with either a liquid-like or a vapor-like phase under confinement.
RPM has been extensively used to represent, among other electrolyte systems, the phase behavior of both room temperature ionic liquids and organic electrolytes. In the former case, the relative permittivity accounts for polarizability effects and other ionic interactions not included in the charged hard-sphere model. In applications of RPM to organic electrolytes, we assume that the solvent is a dielectric continuum with relative permittivity $\epsilon$. At room temperature, the dielectric constants of bulk ionic liquids are approximately in the range of 5-15, ${ }^{37}$ and $\epsilon=5.5$ is chosen in this work to represent a residual relative permittivity for pair interactions between ions. The same dielectric constant is assumed for organic electrolytes or ionic liquids in organic solvents. It should be noted that, for a given ionic system, the relative permittivity is in general not the same as that corresponding to the bulk value; the former accounts for interactions beyond the Coulomb potential between a pair of isolated ions in the medium, while the latter is related to the potential of mean force between ions in a bulk ionic system.

To make RPM relevant to EDL capacitors for energy storage, we take dimensionless temperature $T^{*}=0.05$ for most of our discussions on the pore size and the surface voltage effects on wettability. Approximately, the condition corresponds to an ionic liquid or an organic electrolyte of ion diameter $\sigma=0.5 \mathrm{~nm}$ at temperature $T \approx 300 \mathrm{~K}$ (e.g., ionic liquid 1-ethyl-3-methylimidazolium bis(trifluoromethylsulfonyl)imide(EMIM-TFSI) or ionic solutions of $\mathrm{C}_{18}$ mim- $\mathrm{NTF}_{2}$ in decalin). The dimensionless temperature is lower than that corresponding to the critical temperature for the vapor-liquid equilibrium of the bulk ionic system presented by the equation of state used in this work $T_{c}^{*} \approx 0.072$.

\section{B. Classical DFT}

The basic ideas of classical DFT have been described in detail. $^{29,30}$ From a theoretical perspective, the essential task is to derive an analytical expression for the grand potential $\Omega$, or equivalently, the intrinsic Helmholtz free energy $F$, as a functional of the density profiles of underlying particles. For an inhomogeneous ionic fluid represented by RPM, the grand potential is related to the intrinsic Helmholtz functional via the Legendre transformation

$$
\Omega\left[\rho_{i}(\mathbf{r})\right]=F\left[\rho_{i}(\mathbf{r})\right]+\int d \mathbf{r} \sum_{i} \rho_{i}(\mathbf{r})\left[V_{i}(\mathbf{r})-\mu_{i}\right],
$$

where $\mu_{i}$ and $\rho_{i}(\mathbf{r})$ denote the chemical potential and the density profiles of ionic species $i$, respectively, and $V_{i}(\mathbf{r})$ stands for a one-body external potential. In a slit pore, both the external potential and the ionic density profiles vary only in the $z$-direction, i.e., $V_{i}(\mathbf{r})=V_{i}(z)$ and $\rho_{i}(\mathbf{r})=\rho_{i}(z)$. Equilibrium properties of the system can be obtained by minimizing the grand potential with respect to the ionic density profiles.

A number of classical DFT methods are available to describe the thermodynamic properties of inhomogeneous ionic systems. ${ }^{38,39}$ Different versions of DFT distinguishes from each other in formulating the intrinsic Helmholtz free energy $F$, which is conventionally decomposed into an ideal 
part to account for the particle translational entropy and an excess for inter-particle interactions,

$$
F\left[\rho_{i}(\mathbf{r})\right]=F^{i d}\left[\rho_{i}(\mathbf{r})\right]+F^{e x}\left[\rho_{i}(\mathbf{r})\right] .
$$

For ionic systems considered in this work, the ideal-gas term is known exactly,

$$
\beta F^{i d}\left[\rho_{i}(\mathbf{r})\right]=\sum_{i=+,-} \int d \mathbf{r} \rho_{i}(\mathbf{r})\left[\ln \left(\rho_{i}(\mathbf{r}) \Lambda_{i}^{3}\right)-1\right],
$$

where $\Lambda_{i}$ denotes the thermal wavelength, a parameter immaterial to phase-equilibrium calculations.

Most previous applications of classical DFT to ionic systems are based on an excess intrinsic Helmholtz energy derived from various forms of the mean-spherical approximation (MSA) ${ }^{40}$ While these methods are able to predict ionic density profiles in reasonably good agreement with simulation data, MSA provides a poor description of the phase behavior of ionic fluids in the bulk. It fails to reproduce the existence of the liquid-vapor coexistence from the virial or the compressibility equation of state, and its predictions of the critical temperature and the critical density through the energy route show severe deviations from molecular simulation values. It has been shown that, to represent the phase behavior of bulk systems, the theoretical performance of MSA can be significantly improved by incorporation of association between oppositely charged ions. $^{41,42}$

Considering the discrepancy between the previous DFT predictions $^{31}$ and simulation results ${ }^{8}$ on the effect of the surface potential on capillary evaporation, we propose in this work a new theoretical scheme whereby MSA is used to account for electrostatic correlations, and the thermodynamic perturbation theory (TPT) is used to represent association between oppositely charged ions. Specifically, we incorporate the modified fundamental measure theory (MFMT) to account for the ionic excluded volume effects, ${ }^{43,44}$ the reference fluid density (RFD) perturbation for electrostatic correlations, ${ }^{45}$ and an extension of MFMT for the association free energy. ${ }^{46}$ The excess intrinsic Helmholtz energy can be formally written as

$$
F^{e x}\left[\rho_{i}(\mathbf{r})\right]=F_{h s}^{e x}\left[\rho_{i}(\mathbf{r})\right]+F_{C}^{e x}\left[\rho_{i}(\mathbf{r})\right]+F_{e l}^{e x}\left[\rho_{i}(\mathbf{r})\right]+F_{a s}^{e x}\left[\rho_{i}(\mathbf{r})\right],
$$

where $F_{h s}^{e x}\left[\rho_{i}(\mathbf{r})\right]$ represents the contribution to the free energy functional due to hard-sphere repulsions, $F_{C}^{e x}\left[\rho_{i}(\mathbf{r})\right]$ is the direct Coulomb energy, $F_{e l}^{e x}\left[\rho_{i}(\mathbf{r})\right]$ arises from electrostatic correlations, and $F_{a s}^{e x}\left[\rho_{i}(\mathbf{r})\right]$ is that from ion associations. Explicit expressions for the individual terms in Eq. (8) are given in the Appendix.

Minimizing the grand potential with respect to the ionic density profiles yields the Euler-Lagrange equation,

$$
\rho_{i}(z)=\rho_{i}^{b} \exp \left[\beta \mu_{i}^{e x}-\beta Z_{i} e \psi(z)-\beta \lambda_{i}^{e x}(z)\right],
$$

where $\rho_{i}^{b}$ and $\mu_{i}^{e x}$ stand for the ionic density and the excess chemical potential of species $i$ in the bulk, respectively, $\psi(z)$ represents the local electrical potential, and $\lambda_{i}^{e x}(z)$ is the local one-body potential other than that due to the direct Coulomb interactions. The excess chemical potential of species $i$ in the bulk can be calculated from MSA. The local electrical potential is related to the surface charge density and the direct electrostatic interactions among ionic species

$$
Z_{i} e \psi(z)=V_{i}^{C}+\frac{\delta F_{C}^{e x}}{\delta \rho_{i}(z)} .
$$

The electrical potential can be solved from the local charge density from the Poisson equation,

$$
\nabla^{2} \psi(z)=-\frac{1}{\epsilon \epsilon_{0}} \sum_{i} Z_{i} e \rho_{i}(z)
$$

For the model electrolyte systems discussed in this work, the extra one-body potential can be written as

$$
\lambda_{i}^{e x}(z)=V_{i}^{H W}(z)+\frac{\delta F_{h s}^{e x}}{\delta \rho_{i}(z)}+\frac{\delta F_{e l}^{e x}}{\delta \rho_{i}(z)}+\frac{\delta F_{a s}^{e x}}{\delta \rho_{i}(z)} .
$$

With suitable boundary conditions and an analytical expression for each item in Eq. (12), we can numerically solve for the density profiles from Eq. (9) using the Picard iteration. From the ionic density profiles, we then calculate the average density of all ions in the pore,

$$
\bar{\rho}=\int_{0}^{H} d z \sum_{i} \rho_{i}(z) / H .
$$

In classical DFT calculations, the ionic density profiles are typically obtained by specifying the surface electrical potential rather than the surface charge density. For an ionic liquid confined in a slit pore of fixed width and surface electrical potential, we can calculate the surface charge density from the electroneutrality condition for the entire system,

$$
Q=-\frac{1}{2} \sum_{i} Z_{i} e \int \rho_{i}(z) d z
$$

\section{RESULTS AND DISCUSSIONS}

\section{A. Phase diagram of confined ionic fluids}

We consider first the phase diagram for the model ionic system in slit pores with uncharged walls. Because of the symmetry for cations and anions, the local electric potential, as well as the local charge density, is everywhere zero, i.e., $\psi^{*}(z)=0$ and $q^{*}(z)=0$. In construction of the phase diagram, we calculate the vapor-like and the liquid-like density profiles at different chemical potentials of the ionic species at each temperature. Subsequently, we obtain two curves for the dependence of the grand potential versus the chemical potential, and the crossing point corresponds to the condition of phase coexistence inside the pore.

Figure 2(a) illustrates the dependence of the reduced grand potential, $\beta \Omega$, on the reduced chemical potential of the ionic species, $\beta \mu$, in a $H^{*}=5.0$ pore at two representative temperatures, $T^{*}=0.050$ and 0.0525 . At each temperature, the two curves provide the thermodynamic relations for the diluteand the concentrated-branches of the confined ionic system, and their intersection corresponds to the condition of phase equilibrium, i.e., two ionic density profiles for the coexisting vapor-like and liquid-like phases (or dilute and concentrated electrolytes) inside the pore. By applying the same procedure to different temperatures, we can construct a phase diagram in 

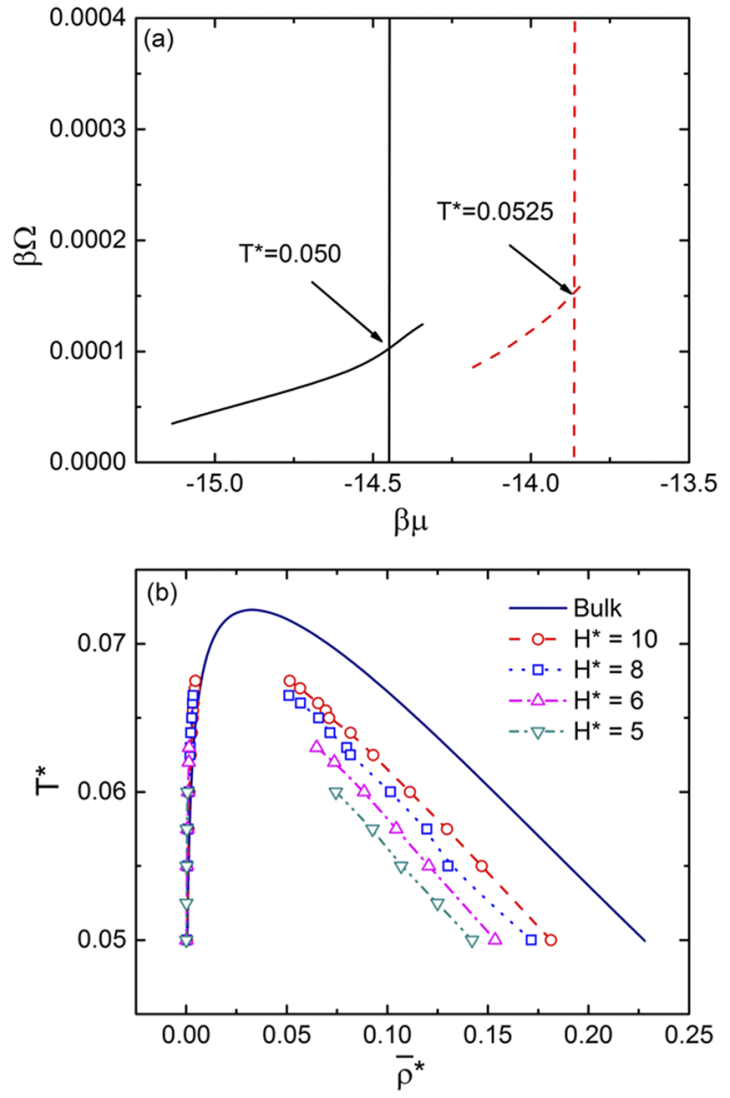

FIG. 2. (a) The reduced grand potential versus the reduced chemical potential of ionic species in a $H=5.0 \sigma$ slit pore. (b) Phase diagram for the ionic fluid in the bulk (solid line) and in different neutral slit pores.

terms of the reduced temperature and the average reduced ion density similar to that for the bulk system.

Figure 2(b) shows the vapor-liquid-like coexistence curves for the model ionic system in slit pores of different pore widths. The solid line corresponds to the coexistence curve in the bulk. Consistent with earlier theoretical investigations, ${ }^{32,47}$ confinement narrows the phase coexistence envelope. While the dilute branch is weakly dependent on the slit pore width, the concentrated branch considerably shifts leftwards as the pore width decreases. Two effects might be responsible for the shift of the binodal curve. First, inhomogeneous screening of electrostatic interactions results in a correlation effect responsible for ion depletion near the surface: ${ }^{48}$ an ion interacts more favorably with its full ionic atmosphere far away from the surface than that in the vicinity of the surface. Second, the leftward shift may also be attributed to the inclusion of association between oppositely charged ions. Pizo and Sokołowski ${ }^{32}$ compared the phase diagram of the ionic system with and without ion associations. They found that formation of pairs between oppositely charged ions alters the effective interactions between all particles. The ion pairing results in a weaker effective attraction between structural entities and thus lowers the critical temperature of the phase transition. In Fig. 2(b), we did not include the critical points for the confined ionic systems due to numerical issues. Nevertheless, it is clear that confinement reduces the critical temperature and the critical density as predicted before. ${ }^{31}$ As the pore width decreases, the entire coexistence curve is shifted toward lower temperatures and lower densities in comparison to the bulk phase diagram.

As shown in Fig. 3, the depletion effect near a neutral wall is evident from the ionic density profiles. For the model ionic system in a neutral slit pore, the density profiles of the cations and anions are identical due to the system symmetry. In a dilute phase, the average ion density is small such that the correlation effect is relatively insignificant. As the ionic density increases, electrostatic correlation becomes more important and ion depletion from the surface is more magnified. Similar to the bulk phase diagram, the average coexisting density of the liquid-like phase falls as temperature increases, while the trend is opposite for the vapor-like phase.

\section{B. Capillary evaporation}

At a given temperature, an ionic fluid in a slit pore may exist either as a liquid-like or as a vapor-like phase depending on the pore size, surface electrical potential, as well as temperature and the chemical potential of the ionic species in the bulk. In this work, capillary evaporation is referred to as the phase transition of the confined fluid from a liquid-like to a vaporlike phase in response to changes in the external potential or thermodynamic conditions.

Figure 4 shows the chemical potential of ionic species in a neutral slit pore and that in the bulk at the condition of vaporliquid-like coexistence. The two curves allow us to predict the wettability of the slit pore in contact with a bulk ionic system at the same temperature and chemical potential. Wetting
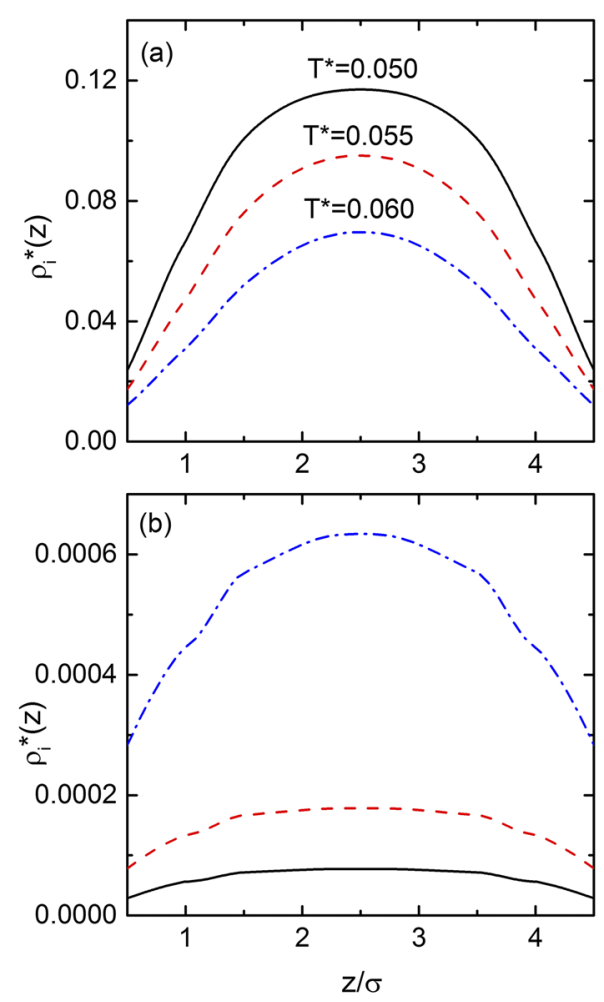

FIG. 3. The density profiles of the liquid-like (a) and vapor-like (b) phases at coexistence for the model ionic system in a neutral slit pore of width $H^{*}=5.0$ at $T^{*}=0.050$ (solid line), 0.055 (dashed line), and 0.060 (dotteddashed line). Because of the symmetry, the density profiles for the cations and anions are identical. 


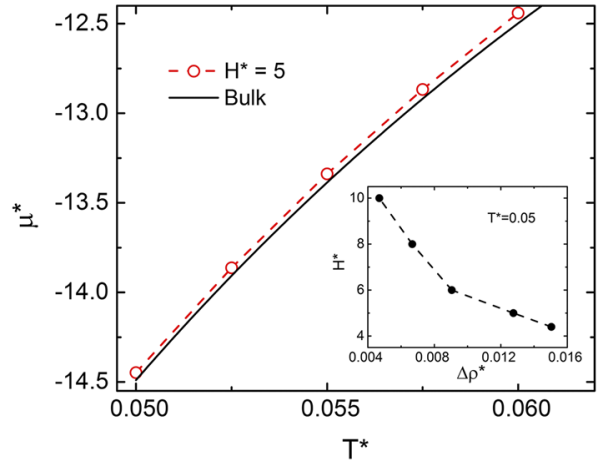

FIG. 4. The $\mu^{*}-T^{*}$ phase diagram for the model ionic system in the bulk and in a $5.0 \sigma$ slit pore. The inset shows the degree of supersaturation versus the pore width at $T^{*}=0.05$. In all cases, the pore surface is uncharged.

transition occurs when the chemical potential of the confined fluid is lower than that in the bulk. As a result, Fig. 4 predicts that a neutral pore promotes capillary evaporation, i.e., the ionic fluid may exist as a stable liquid in the bulk but a vapor-like phase inside the pore.

The inset of Fig. 4 shows the dependence of the pore width on the "degree of supersaturation" defined as $\Delta \rho^{*}=\rho_{b, c o}^{*}\left(H^{*}\right)-\rho_{b}^{*}$, where $\rho_{b}^{*}$ is the density of the bulk liquid at saturation and $\rho_{b, c o}^{*}\left(H^{*}\right)$ is the density of a bulk liquid that has a chemical potential the same as that of the saturated liquid inside a pore of width $H^{*} . \rho_{b, c o}^{*}\left(H^{*}\right)$ can be obtained from the phase diagram shown in Fig. 2. Figure 4 suggests that, to make the liquid state stable inside the pore or to make the pore wet, the corresponding ionic system in the bulk should be "supersaturated," i.e., the ionic density should be larger than that of a saturated liquid at the same temperature. Otherwise, capillary evaporation (or dewetting) takes place inside the pore. For example, for a given ionic liquid with $\Delta \rho^{*} \approx 0.009$, the coexistence of the liquid-like and vapor-like phases occurs near $H_{c}^{*} \approx 6.0$. In a larger pore, such as $H^{*}=10.0$, only the liquidlike state is stable; below $H_{c}^{*}$, the liquid-like state becomes metastable in comparison with the vapor-like phase, and the phase transition becomes unavoidable.

Capillary evaporation of ionic liquids in porous carbons has been observed in both experimental and simulation studies. For example, Gogotsi et al. ${ }^{49}$ showed that 1-ethyl-3-methylimidazolium bis(trifluoromethylsulfonyl) imide ([EMIM][TFSI]) cannot wet micropores smaller than $0.75 \mathrm{~nm}$ at zero applied potential. Shrivastav and co-workers found from MD simulations a critical pore width, $d_{c} \approx 1.2 \mathrm{~nm}$, for [EMIM] $\left[\mathrm{BF}_{4}\right]$ in contact with neutral pores. ${ }^{11}$ While a quantitative comparison of the theoretical predictions with the experimental or simulation results has not been attempted in this study, it is clear that small pores may not be accessible to an ionic fluid due to dewetting or capillary evaporation.

\section{Electrowetting and differential capacitance}

Now we proceed to investigate the wettability of the model ionic system in charged pores. Figure 5(a) shows the surface charge density as a function of the applied voltage for a slit pore of width $H=3.5 \sigma$ in contact with a bulk ionic fluid at three representative densities. Because of the symmetry of the ionic system, the dependence of the surface charge

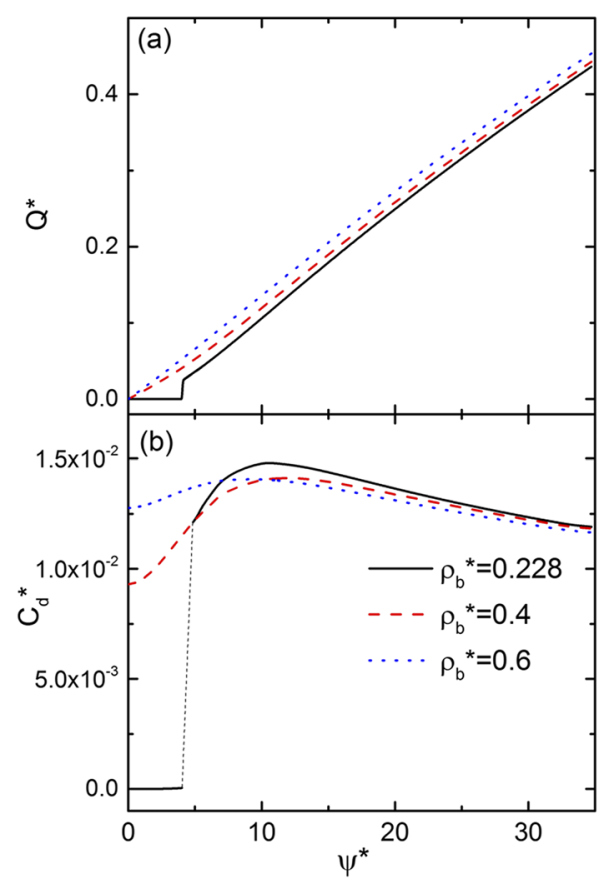

FIG. 5. The dependence of surface charge density $Q^{*}$ (a) and the differential capacitance $C_{d}^{*}$ (b) on the applied voltage. The calculations are for different bulk densities given in (b). In all cases, the pore width is $H^{*}=3.5$, and the reduced temperature is $T^{*}=0.05$. The reduced voltage and charge density are $\psi^{*}=\beta \psi e$ and $Q^{*}=Q \sigma^{2} / e$, respectively.

density on the surface voltage is equally applicable to conditions when both quantities are negative. As the surface voltage of the slit pore increases, the surface charge increases smoothly from zero at high ionic densities (i.e., $\rho_{b}^{*}=0.4$ and 0.6). In this case, the slit pore is always filled with a liquid-like ionic fluid because the density is remote from that near the vapor-liquid-like coexistence. At a lower density $\left(\rho_{b}^{*}=0.228\right)$, however, the surface charge density is virtually zero for a slit pore with a small surface potential; it increases discontinuously to a finite value when the surface potential is above around $\psi^{*} \approx 4.1$, signaling a first-order phase transition inside the pore. Qualitatively, the discontinuous variation of the surface charge density in the $\psi^{*}-Q^{*}$ phase diagram is consistent with that predicted by Kiyohara and co-workers using Monte Carlo simulation. ${ }^{8}$ The phase transition can be understood as a manifestation of the balance between the electrostatic correlations and the volume exclusion interactions. While the electrostatic correlations lead to depletion of the ionic fluid from a neutral surface, application of a surface potential results in electrowetting because of the accumulation of counterions.

A key quantity of practical interest for energy storage is the differential capacitance, which is defined as

$$
C_{d}^{*}=\partial Q^{*} / \partial \psi^{*} .
$$

Without an explicit consideration of phase transitions, the differential capacitance is always a smooth function of the surface voltage as predicted by typical EDL models.

Figure 5(b) shows how the differential capacitance varies with the applied surface voltage for the ionic systems described above. At high ionic density (e.g., $\rho_{b}^{*}=0.4$ ), the $\psi^{*}-C_{d}^{*}$ curve exhibits a camel-shape, showing a minimum at zero surface 
voltage and a maximum at a higher surface voltage. Increasing the density from $\rho_{b}^{*}=0.4$ to 0.6 , the $\psi^{*}-C_{d}^{*}$ curve still shows a camel-shape, while the differential capacitance at zero voltage increases. As discussed in previous studies, ${ }^{50-52}$ a further increase in the bulk density induces the change of the $\psi^{*}-C_{d}^{*}$ curve from the camel-shape to a bell-shape. For the model ionic liquid at a lower density, $\rho_{b}^{*}=0.228$, the $\psi^{*}-C_{d}^{*}$ curve shows a discontinuity around $\psi^{*} \approx 4.1$, at which condition the first-order phase transition is observed.

Figure 6 presents the density profiles of ions inside the pore at surface potentials slightly below and above that corresponding to the jump in the surface charge density. At $\psi^{*}=4.2$, the ionic fluid is in a liquid-like state inside the pore. Although counterions are accumulated near the surface, the local density inside the pore is uniformly lower than that in the bulk due to the depletion of the coions. At the pore center, the coion concentration is slightly larger than the counterion concentration, which can be attributed to the local charge inversion, i.e., the local electrical potential has a sign opposite to that of the surface charge. At a slightly lower surface potential, $\psi^{*}=4.0$, the pore is filled with an ionic fluid at a much lower density. In this case, the density profiles show depletion of both coions and counterions because of long-range electrostatic correlations. As expected, $C_{d}^{*}$ is virtually negligible if the micropore is filled with a lowdensity ionic fluid. A drastic increase in the capacitance occurs when the slit pore is wetted by the ionic fluid, i.e., when the ionic fluid inside the pore exists in a liquid-like state [see Fig. 5(b)].

Figure 7(a) shows the surface charge density as a function of the voltage for different pore sizes, $H^{*}=1.6,2.0$, $2.4,3.2$, and 4.8. In small pores (e.g., $H^{*}<4.8$ ), the surface charge density is virtually zero until the voltage is larger

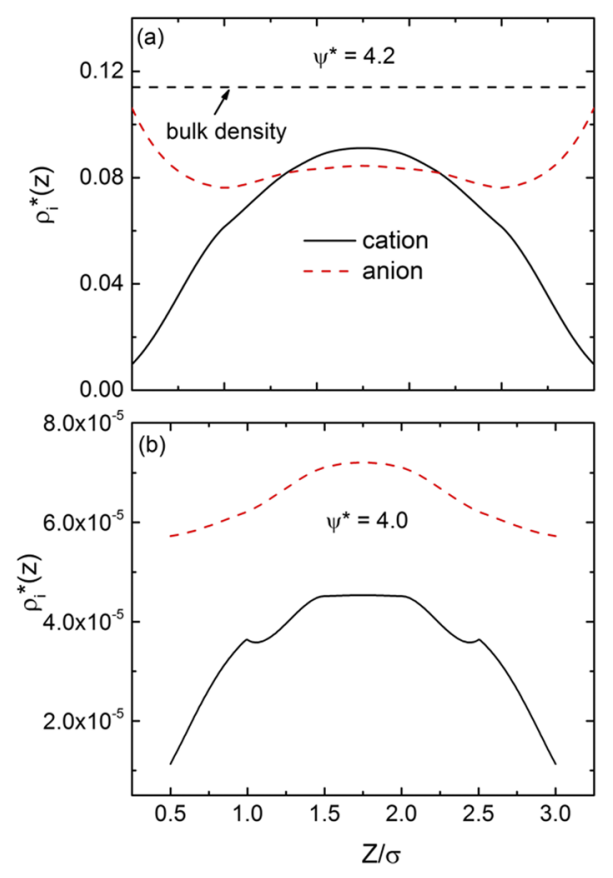

FIG. 6. Density profiles of cations and anions in a slit pore of $H=3.5 \sigma$ at $T^{*}=0.05$ and reduced surface potential (a) $\psi^{*}=4.2$ and (b) $\psi^{*}=4.0$. The reduced density for the bulk ionic liquid is $\rho_{b}^{*}=0.228$.
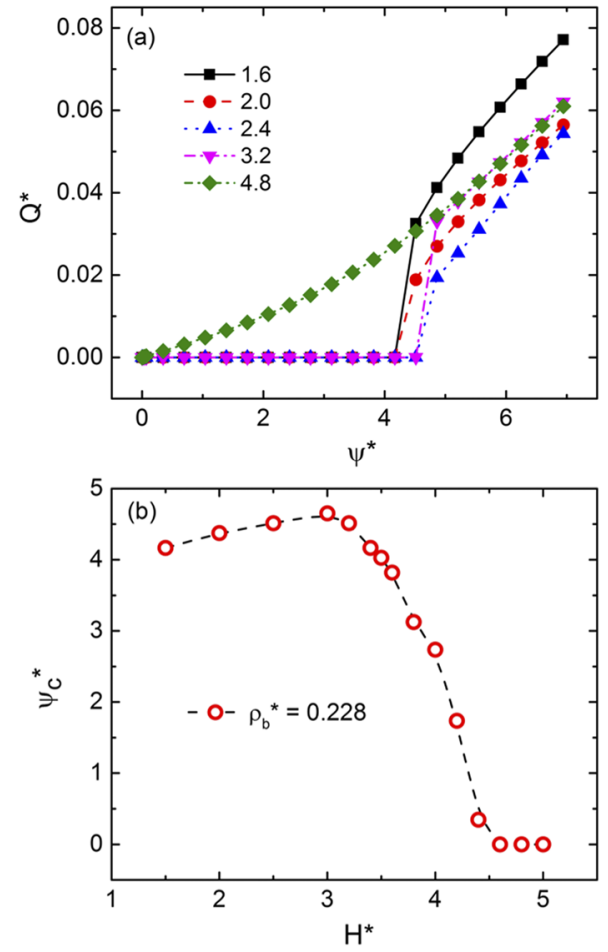

FIG. 7. (a) The surface charge density as a function of the voltage in slit pores of different reduced pore width $H^{*}=H / \sigma$. (b) The critical surface potential as a function of the reduced pore width. Here the density for the bulk ionic liquid is $\rho_{b}^{*}=0.228$.

than a certain value, $\psi^{*} \approx 4.0$. Beyond that voltage, the surface charge density jumps to a finite value and rises continuously as the voltage is further increased. In a large pore (viz., $H^{*}=4.8$ ), the surface charge density increases smoothly with the surface potential over the entire range of the voltage. Qualitatively, the response of the surface charge density to the surface potential is similar to the MC results reported by Kiyohara ${ }^{8}$ but contradicts to that from earlier classical DFT predictions. ${ }^{31}$ Although MSA was used in both versions of classical DFT, the results are qualitatively different because we formulated the free-energy functional in terms of the non-local reference fluid theory and the thermodynamic perturbation theory rather than the weighted density approximations. Apparently, the subtle difference leads to opposite predictions of the phase behavior of the confined ionic fluids.

The surface voltage at which the discontinuity of differential capacitance occurs can be regarded as the "critical" voltage, $\psi_{c}^{*}$. Beyond this voltage, the slit pore is filled with an ionic fluid in the liquid-like state. Figure 7(b) shows the dependence of the critical voltage as a function of the pore size when the ion density is approaching that for the saturated liquid in the bulk. $\psi_{c}^{*}$ disappears for $H^{*}>4.5$, suggesting that the first-order phase transition occurs only in small pores. Interestingly, the critical voltage first increases slightly with the pore size and falls abruptly beyond a certain pore width. The non-monotonic behavior reflects not only a competition of electrostatic correlations and excluded-volume interactions but also the confinement effects on the vapor-liquid-like transitions. 


\section{CONCLUSION}

To conclude, we have investigated the phase behavior of ionic fluids in the micropores of carbon electrodes using the restricted primitive model (RPM). By constructing a new version of the classical density functional theory (DFT) that accounts for electrostatic correlations and associating between oppositely charged ions, we demonstrate that capillary evaporation may take place in the micropores of carbon when the pore size is comparable to the ion diameter of nonaqueous electrolytes and that application of a surface electrical potential promotes wetting transition. In the latter case, the theoretical results contradict earlier DFT predictions but are consistent with recent Monte Carlo simulation, suggesting that the DFT performance is sensitive to the formulation of the free-energy functional, in particular, for phase-equilibrium calculations.

In stark contrast to conventional understandings of the accessibility of micropores to ionic fluids, capillary evaporation is determined by the pore geometry as well as ion-ion and ion-surface interactions underlying the phase behavior of the entire ionic system. Because capillary evaporation may result in a drastic reduction in the capacitance, the pore size effects on the performance of carbon electrodes depend on the temperature, the ionic density, as well as the surface electrical potential. According to our theoretical calculations, wetting transition is most likely due to the liquid-liquid phase separation of an organic electrolyte in small pores. Vapor-liquid-like coexistence is less likely for room temperature ionic liquids because the liquid density is typically remote from that corresponding to the coexistence point. The sharp difference may help explain discrepancies observed in experiments on the pore effects on the performance of various carbon electrodes for capacitive energy storage.

RPM is clearly oversimplified to quantitatively represent the properties of real ionic liquids or organic electrolytes. Nevertheless, it is our hope that this work would usher in a new direction of theoretical investigation of electrolytes in a porous electrode beyond electric double layer models that have been of central interest in electrochemistry for centuries. To highlight the essential features of the capillary evaporation induced by ion-ion correlation and confinement, we have implicitly assumed in this work that the dielectric constant of the electrode is the same as that of the electrolyte solution. In experiment, the dielectric constant of the electrode and that of the solution are generally different and this dielectric discontinuity may also play a significant role in determining the surface structure, as suggested by recent theoretical and simulation work. ${ }^{18,53-55}$ A systematic study on all of these effects, however, is still lacking and will be pursued in future work.

\section{ACKNOWLEDGMENTS}

This work was supported as part of the Fluid Interface Reactions, Structures and Transport (FIRST) Center, an Energy Frontier Research Center funded by the U.S. Department of Energy, Office of Science, Office of Basic Energy Sciences. Some calculations in this work are performed on the National Energy Research Scientific Computing (NERSC)
Center. The authors would like to thank Dr. Jian Jiang for helpful discussions.

\section{APPENDIX: SUPPLEMENTAL MATERIAL}

Here we present detail expressions for different contributions to the excess Helmholtz energy functional and the corresponding equations for the local excess chemical potential of ions for the model ionic system (RPM) considered in this work.

\section{Hard sphere term}

The excess free energy due to the hard-sphere repulsion is represented by ${ }^{43,44}$

$$
\beta F_{h s}^{e x}\left[\rho_{i}(\mathbf{r})\right]=\int d \mathbf{r} \Phi^{h s}\left[n_{\alpha}(\mathbf{r})\right],
$$

where the reduced excess Helmholtz energy density $\Phi^{h s}$ is a function of the weighted averages of the density distribution functions $\rho_{i}(\mathbf{r})$,

$$
n_{\alpha}(\mathbf{r})=\sum_{\mathbf{i}} n_{\alpha, i}(\mathbf{r})=\sum_{\mathbf{i}} \int d \mathbf{r} \rho_{\mathbf{i}}\left(\mathbf{r}^{\prime}\right) \omega_{\mathbf{i}}^{(\alpha)}\left(\mathbf{r}-\mathbf{r}^{\prime}\right)
$$

The weight functions $\omega_{i}^{(\alpha)}$ in Eq. (A2) characterize the geometry of each hard sphere: the volume, the surface area, and the mean radius of curvature. The detailed expression for the weighted densities, $n_{\alpha}(\mathbf{r}), \alpha=0,1,2,3, V 1, V 2$, can be found in our previous publications or in Rosenfeld's original work. ${ }^{43}$ The final expression for the excess Helmholtz energy density is given by

$$
\begin{aligned}
\Phi^{h s}= & -n_{0} \ln \left(1-n_{3}\right)+\frac{n_{1} n_{2}-\mathbf{n}_{V 1} \mathbf{n}_{V 2}}{1-n_{3}}+\left(n_{2}^{3}-3 n_{2} \mathbf{n}_{V 2} \mathbf{n}_{V 2}\right) \\
& \times\left[\frac{n_{3}+\left(1-n_{3}\right)^{2} \ln \left(1-n_{3}\right)}{36 \pi n_{3}^{2}\left(1-n_{3}\right)^{2}}\right] .
\end{aligned}
$$

In the bulk limit, the two vector weighted densities $\mathbf{n}_{V 1}$ and $\mathbf{n}_{V 2}$ vanish and $\Phi^{h s}$ becomes identical to that from the BoublikMansoori-Carnahan-Starling-Lelandc (BMCSL) equation of state. ${ }^{56}$

The functional derivative of excess Helmholtz free energy due to the hard-sphere yields the excess chemical potential $\mu_{h s, i}^{e x}$ given by

$$
\beta \mu_{h s, i}^{e x}(\mathbf{r})=\frac{\delta \beta F_{h s}^{e x}}{\delta \rho_{i}(\mathbf{r})}=\sum_{\alpha} \int d \mathbf{r}^{\prime} \frac{\partial \Phi_{h s}^{e x}}{\partial n_{\alpha}\left(\mathbf{r}^{\prime}\right)} \omega_{i}^{(\alpha)}\left(\mathbf{r}^{\prime}-\mathbf{r}\right) .
$$

\section{Direct coulomb term}

The Helmholtz energy due to the direct Coulomb potential is given by

$$
\beta F_{C}^{e x}\left[\rho_{i}(\mathbf{r})\right]=\frac{l_{B}}{2} \sum_{i, j} \iint d \mathbf{r} d \mathbf{r}^{\prime} \frac{Z_{i} Z_{j} \rho_{i}(\mathbf{r}) \rho_{j}\left(\mathbf{r}^{\prime}\right)}{\left|\mathbf{r}-\mathbf{r}^{\prime}\right|} .
$$

The excess chemical potential due to direct coulomb interaction $\mu_{C, i}^{e x}(\mathbf{r})$ is often calculated from the local mean electrostatic potential $\psi(\mathbf{r})$ by Eqs. (10) and (11). To be noted is that the excess chemical potential in bulk vanishes because of charge neutrality in bulk electrolyte solution. 


\section{Electrostatic correlation term}

For the electrostatic correlation term, we followed the work of Gillespie and co-workers, ${ }^{45}$ in which the positiondependent reference fluid densities (RFD) are introduced to define a "smoothed," nonuniform fluid as the reference state instead of the bulk fluid. By using this method, the local charge neutrality is satisfied everywhere and the density profile is in good agreement with the simulation results for the fluid near a uncharged or low-charged wall, ${ }^{57}$

$$
\begin{aligned}
\beta F_{e l}^{e x}\left[\rho_{i}(\mathbf{r})\right] \approx & \beta F_{e l}^{e x}\left[\rho_{i}^{\mathrm{ref}}(\mathbf{r})\right]-\sum_{i} \int c_{i}^{(1), e l} \Delta \rho_{i}(\mathbf{r}) d \mathbf{r} \\
& -\frac{1}{2} \sum_{i, j} \iint c_{i, j}^{(2), e l} \Delta \rho_{i}(\mathbf{r}) \Delta \rho_{j}\left(\mathbf{r}^{\prime}\right) d \mathbf{r} d \mathbf{r}^{\prime},
\end{aligned}
$$

where $\rho_{i}^{\text {ref }}(\mathbf{r})$ is the ionic density profiles of a locally defined reference fluid and $\Delta \rho_{i}(\mathbf{r})=\rho_{i}(\mathbf{r})-\rho_{i}^{\text {ref }}(\mathbf{r}) . c_{i}^{(1), e l}$ and $c_{i j}^{(2), e l}$ are the first- and second-order DCFs, respectively. The detailed expression for $\rho_{i}^{\text {ref }}(\mathbf{r})$ can be found in the original publication. ${ }^{45}$ Because local charge neutrality is everywhere satisfied in the reference system, $c_{i}^{(1), e l}$ and $c_{i j}^{(2), e l}$ can also be calculated from MSA for a bulk electrolyte. ${ }^{38}$ The excess chemical potential due to electrostatic correlations is given by

$$
\beta \mu_{i}^{e l}(\mathbf{r}) \approx \beta \mu_{i}^{e l}\left[\rho_{i}^{r e f}(\mathbf{r})\right]-\sum_{j} \int d \mathbf{r}^{\prime} c_{i, j}^{(2), e l} \Delta \rho_{j}\left(\mathbf{r}^{\prime}\right) .
$$

\section{Ion association term}

The Helmholtz energy due to association between oppositely charged ions is formulated at the level of the first-order thermodynamic perturbation (TPT1) theory, ${ }^{46}$

$$
\beta F_{a s}^{e x}\left[\rho_{i}(\mathbf{r})\right]=\int d \mathbf{r} \Phi^{a s}\left[n_{\alpha}(\mathbf{r})\right],
$$

where $\Phi^{a s}\left[n_{\alpha}(\mathbf{r})\right]$ is given by

$$
\Phi^{a s}\left(n_{\alpha}\right)=\sum_{i} n_{0, i} \zeta_{i}\left[\ln \alpha^{(i)}(\mathbf{r})-\frac{\alpha^{(i)}(\mathbf{r})}{2}+\frac{1}{2}\right] .
$$

Similar to that for association in uniform systems, $\alpha^{(i)}(\mathbf{r})$ is the degree of dissociation of species $i$ at position $\mathbf{r}$, and it is obtained from

$$
\alpha^{(i)}(\mathbf{r})=\frac{1}{1+n_{0 j} \zeta_{j} \alpha^{(j)}(\mathbf{r}) \Delta^{i j}(\mathbf{r})},
$$

where $\Delta^{i j}(\mathbf{r})$ is the association constant between cations and anions, $\Delta^{i j}(\mathbf{r})=K^{0} \times K^{\gamma}$.

There is a certain kind of arbitrariness in defining the ion pair and hence the association constant $K^{0}$. Among several definitions of the ion-association constant, the one introduced by Ebling yields the correct second ionic-virial coefficient. ${ }^{58}$ However, this approach does not produce good values for the critical temperature and the critical density of the RPM. As discussed in Ref. 47 , we choose $K^{0}$ in the form proposed by Olaussen and Stell, ${ }^{59}$

$$
K^{0} \approx 96 \pi \sigma^{3} \sum_{m=2}^{\infty} \frac{\left(T^{*}\right)^{-2 m}}{(2 m) !(2 m-3)} .
$$

$K^{\gamma}$ is calculated from the simple interpolation scheme

$$
K^{\gamma}=y_{+-}(\sigma)
$$

where $y_{+-}(\sigma)$ is the contact anion-cation cavity correlation function evaluated at $\alpha=1$, the reference ionic fluid without association. As a result, $y_{+-}(\sigma)$ is given by

$$
\begin{aligned}
y_{+-}(\sigma)= & {\left[\frac{1}{1-n_{3}}+\frac{n_{2} \sigma_{+} \sigma_{-}\left(1-\mathbf{n}_{V 2} \mathbf{n}_{V 2} / \mathbf{n}_{2}^{2}\right)}{4 \sigma_{+-}\left(1-n_{3}\right)^{2}}\right] } \\
& \times \exp \left(-\frac{\Gamma^{2} a_{+} a_{-}}{4 \pi^{2} l_{B} \sigma_{+-}}\right) \exp \left(\frac{l_{B} Z_{+} Z_{-}}{\sigma_{+-}}\right) .
\end{aligned}
$$

Similar to the thermodynamic perturbation theory for bulk solutions, ${ }^{60,61}$ parameters $\Gamma$ and $a_{k}$ can be estimated by following a simple iterative procedure,

$$
\begin{gathered}
\Gamma^{2}=\pi l_{B} \sum_{i} \frac{n_{0 i}}{\left(1+\Gamma \sigma_{i}\right)^{2}}\left[Z_{i}-\frac{\pi P_{n} \sigma_{i}^{2}}{2\left(1-n_{3}\right)}\right]^{2}, \\
a_{i}=\frac{2 \pi l_{B}\left(Z_{i}-\frac{\pi P_{n} \sigma_{i}^{2}}{2\left(1-n_{3}\right)}\right)}{\Gamma\left(1+\Gamma \sigma_{i}\right)},
\end{gathered}
$$

with

$$
P_{n}=\sum_{i} \frac{2 n_{1 i} Z_{i}}{1+\Gamma \sigma_{i}} /\left[1+\frac{3}{\left(1-n_{3}\right)} \sum_{i} \frac{n_{3 i}}{1+\Gamma \sigma_{i}}\right] .
$$

The functional derivative of excess Helmholtz free energy due to the ion association yields the excess chemical potential $\mu_{a s}^{e x}(\mathbf{r})$ given by

$\beta \mu_{a s, i}^{e x}(\mathbf{r})=\frac{\delta \beta F_{a s}^{e x}}{\delta \rho_{i}(\mathbf{r})}=\sum_{\alpha} \int d \mathbf{r}^{\prime} \frac{\partial \Phi_{a s}^{e x}}{\partial n_{\alpha}\left(\mathbf{r}^{\prime}\right)} \omega_{i}^{(\alpha)}\left(\mathbf{r}^{\prime}-\mathbf{r}\right)$.

${ }^{1}$ C. Liu, X. Yan, F. Hu, G. Gao, G. Wu, and X. Yang, Adv. Mater. 30, 1705713 (2018).

${ }^{2}$ C. Zhan, C. Lian, Y. Zhang, M. W. Thompson, Y. Xie, J. Wu, P. R. Kent, P. T. Cummings, D.-e. Jiang, and D. J. Wesolowski, Adv. Sci. 4, 1700059 (2017).

${ }^{3}$ M. V. Fedorov and A. A. Kornyshev, Chem. Rev. 114, 2978 (2014).

${ }^{4}$ D.-e. Jiang and J. Wu, J. Phys. Chem. Lett. 4, 1260 (2013).

${ }^{5}$ A. Fuertes, G. Lota, T. Centeno, and E. Frackowiak, Electrochim. Acta 50, 2799 (2005).

${ }^{6}$ N. Jackel, P. Simon, Y. Gogotsi, and V. Presser, ACS Energy Lett. 1, 1262 (2016).

${ }^{7}$ S. Kondrat, P. Wu, R. Qiao, and A. A. Kornyshev, Nat. Mater. 13, 387 (2014).

${ }^{8}$ K. Kiyohara, T. Sugino, and K. Asaka, J. Chem. Phys. 134, 154710 (2011).

${ }^{9}$ K. Kiyohara, H. Shioyama, T. Sugino, and K. Asaka, J. Chem. Phys. 136, 094701 (2012).

${ }^{10}$ F. J. M. Ruiz-Cabello, P. Maroni, and M. Borkovec, J. Chem. Phys. 138, 234705 (2013).

${ }^{11}$ G. Shrivastav, R. C. Remsing, and H. K. Kashyap, J. Chem. Phys. 148, 193810 (2018).

${ }^{12}$ Y. Tian and J. Wu, AIChE J. 64, 286 (2018).

${ }^{13}$ A. García-Gómez, G. Moreno-Fernández, B. Lobato, and T. A. Centeno, Phys. Chem. Chem. Phys. 17, 15687 (2015).

${ }^{14}$ K. Urita, C. Urita, K. Fujita, K. Horio, M. Yoshida, and I. Moriguchi, Nanoscale 9, 15643 (2017).

${ }^{15}$ D.-e. Jiang, Z. Jin, and J. Wu, Nano Lett. 11, 5373 (2011).

${ }^{16} \mathrm{P}$. Wu, J. Huang, V. Meunier, B. G. Sumpter, and R. Qiao, ACS Nano 5, 9044 (2011).

${ }^{17}$ G. Feng and P. T. Cummings, J. Phys. Chem. Lett. 2, 2859 (2011).

${ }^{18}$ S. Kondrat and A. Kornyshev, J. Phys.: Condens. Matter 23, 022201 (2010).

${ }^{19}$ S. Boukhalfa, D. Gordon, L. He, Y. B. Melnichenko, N. Nitta, A. Magasinski, and G. Yushin, ACS Nano 8, 2495 (2014). 
${ }^{20}$ D. Bonn and D. Ross, Rep. Prog. Phys. 64, 1085 (2001).

${ }^{21}$ L. D. Gelb, K. Gubbins, R. Radhakrishnan, and M. Sliwinska-Bartkowiak, Rep. Prog. Phys. 62, 1573 (1999).

${ }^{22}$ G. Stell, J. Stat. Phys. 78, 197 (1995).

${ }^{23}$ Y. Levin, Rep. Prog. Phys. 65, 1577 (2002).

${ }^{24}$ H. Lu, B. Li, S. Nordholm, C. E. Woodward, and J. Forsman, J. Chem. Phys. 145, 234510 (2016).

${ }^{25}$ M. Wagner, O. Stanga, and W. Schröer, Phys. Chem. Chem. Phys. 6, 4421 (2004).

${ }^{26}$ W. Schröer and V. Vale, J. Phys.: Condens. Matter 21, 424119 (2009).

${ }^{27}$ R. Szparaga, C. E. Woodward, and J. Forsman, J. Phys. Chem. C 117, 1728 (2013).

${ }^{28}$ M. Mußotter and M. Bier, Phys. Rev. E 96, 032605 (2017).

${ }^{29}$ R. Evans, Adv. Phys. 28, 143 (1979).

${ }^{30}$ J. Wu and Z. Li, Annu. Rev. Phys. Chem. 58, 85 (2007).

${ }^{31}$ O. Pizio, A. Patrykiejew, and S. Sokołowski, J. Chem. Phys. 121, 11957 (2004).

${ }^{32}$ O. Pizio and S. Sokołowski, J. Chem. Phys. 122, 144707 (2005).

${ }^{33}$ B. Loubet, M. Manghi, and J. Palmeri, J. Chem. Phys. 145, 044107 (2016).

${ }^{34}$ M. Holovko, O. Patsahan, and T. Patsahan, J. Phys.: Condens. Matter 28, 414003 (2016).

${ }^{35}$ M. Holovko, T. Patsahan, and O. Patsahan, J. Mol. Liq. 235, 53 (2017).

${ }^{36}$ K. Kiyohara, H. Shioyama, T. Sugino, K. Asaka, Y. Soneda, K. Imoto, and M. Kodama, J. Chem. Phys. 138, 234704 (2013).

${ }^{37}$ T. Singh and A. Kumar, J. Phys. Chem. B 112, 12968 (2008).

${ }^{38}$ J. Jiang, D. Cao, D. Henderson, and J. Wu, J. Chem. Phys. 140, 044714 (2014).

${ }^{39}$ R. Roth and D. Gillespie, J. Phys.: Condens. Matter 28, 244006 (2016).

${ }^{40}$ L. Blum, Mol. Phys. 30, 1529 (1975).
${ }^{41}$ J. Jiang, L. Blum, O. Bernard, J. M. Prausnitz, and S. I. Sandler, J. Chem. Phys. 116, 7977 (2002).

${ }^{42}$ Y. Qin and J. M. Prausnitz, J. Chem. Phys. 121, 3181 (2004).

${ }^{43}$ Y.-X. Yu and J. Wu, J. Chem. Phys. 116, 7094 (2002).

${ }^{44}$ R. Roth, R. Evans, A. Lang, and G. Kahl, J. Phys.: Condens. Matter 14, 12063 (2002).

${ }^{45}$ D. Gillespie, W. Nonner, and R. S. Eisenberg, J. Phys.: Condens. Matter 14, 12129 (2002).

${ }^{46}$ Y.-X. Yu and J. Wu, J. Chem. Phys. 117, 10156 (2002).

${ }^{47}$ M. Holovko, T. Patsahan, and O. Patsahan, J. Mol. Liq. 228, 215 (2017).

${ }^{48}$ R. Wang and Z.-G. Wang, J. Chem. Phys. 142, 104705 (2015).

${ }^{49}$ J. Vatamanu, Z. Hu, D. Bedrov, C. Perez, and Y. Gogotsi, J. Phys. Chem. Lett. 4, 2829 (2013).

${ }^{50}$ A. A. Kornyshev, J. Phys. Chem. B 111, 5545 (2007).

${ }^{51}$ M. T. Alam, M. M. Islam, T. Okajima, and T. Ohsaka, Electrochem. Commun. 9, 2370 (2007).

${ }^{52}$ D.-e. Jiang, D. Meng, and J. Wu, Chem. Phys. Lett. 504, 153 (2011).

${ }^{53}$ R. Wang and Z.-G. Wang, J. Chem. Phys. 139, 124702 (2013).

${ }^{54}$ A. A. Lee and S. Perkin, J. Phys. Chem. Lett. 7, 2753 (2016).

${ }^{55}$ M. Girotto, A. P. dos Santos, and Y. Levin, J. Chem. Phys. 147, 074109 (2017).

${ }^{56}$ G. Mansoori, N. F. Carnahan, K. Starling, and T. Leland, Jr., J. Chem. Phys. 54, 1523 (1971).

${ }^{57}$ D. Gillespie, M. Valiskó, and D. Boda, J. Phys.: Condens. Matter 17, 6609 (2005).

${ }^{58}$ W. Ebeling and M. Grigo, J. Solution Chem. 11, 151 (1982).

${ }^{59}$ K. Olaussen and G. Stell, J. Stat. Phys. 62, 221 (1991).

${ }^{60}$ J. Jiang, L. Blum, O. Bernard, and J. M. Prausnitz, Mol. Phys. 99, 1121 (2001).

${ }^{61}$ Z. Li and J. Wu, J. Phys. Chem. B 110, 7473 (2006). 